\title{
Analysis of the triply heavy baryon states with the QCD sum rules
}

\author{
Zhi-Gang Wang
}

\begin{abstract}
In this article, we reexamine the mass spectrum of the ground state triply heavy baryon states with the QCD sum rules by carrying out the operator product expansion up to the vacuum condensates of dimension 6 in a consistent way and preforming a novel analysis. It is for the first time to take into account the three-gluon condensates in the QCD sum rules for the triply heavy baryon states.
\end{abstract}

Keywords: Triply heavy baryon states, QCD sum rules

PACS number: $12.39 . \mathrm{Mk}, 12.38 . \mathrm{Lg}$

\section{Introduction}

In recent years, a large number of heavy baryon states, charmonium-like states and bottomonium-like states have been observed, which have attracted intensive attentions and have revitalized many works on the singly heavy, doubly heavy, triply heavy and quadruply heavy hadron spectroscopy [1]. In 2017, the LHCb collaboration observed the doubly charmed baryon state $\Xi_{c c}^{++}$in the $\Lambda_{c}^{+} K^{-} \pi^{+} \pi^{+}$mass spectrum for the first time [2], while the doubly charmed baryon states $\Xi_{c c}^{+}$and $\Omega_{c c}^{+}$are still unobserved. In 2020, the LHCb collaboration studied the $J / \psi J / \psi$ invariant mass distribution using $p p$ collision data at center-of-mass energies of $\sqrt{s}=7,8$ and $13 \mathrm{TeV}$ and observed a narrow resonance structure $X(6900)$ around $6.9 \mathrm{GeV}$ and a broad structure just above the $J / \psi J / \psi$ mass with global significances of more than $5 \sigma$ [3]. They are good candidates for the fully charmed tetraquark states and are the first fully heavy exotic multiquark candidates claimed experimentally to date. If they are really fully charmed tetraquark states, we have observed doubly charmed and quadruply charmed hadrons, but we find no experimental evidences for the triply charmed

Correspondence: zgwang@aliyun.com

Department of Physics, North China Electric Power University, Baoding 071003, People's Republic of China hadrons. The observation of the $\Xi_{c c}^{++}$and $X(6900)$ provides some crucial experimental inputs on the strong correlation between the two charm quarks, which may shed light on the spectroscopy of the doubly heavy, triply heavy baryon states, doubly heavy, triply heavy, quadruply heavy tetraquark states, and pentaquark states. On the other hand, the spectrum of the triply heavy baryon states have been studied extensively via different theoretical approaches, such as the lattice QCD [4-11], the QCD sum rules [12-15], various potential quark models [1631], Fadeev equation [32-35], and the Regge trajectories $[36,37]$. The predicted triply heavy baryon masses vary in a rather large range. More theoretical works are still needed to obtain more precise inputs for comparing to the experimental data in the future.

The QCD sum rules approach is a powerful theoretical tool in studying the mass spectrum of the heavy flavor hadrons and plays an important role in assigning the new baryon states, exotic tetraquark (molecular) states, and pentaquark (molecular) states. The ground state triply heavy baryon states $Q Q Q$ and $Q Q Q^{\prime}$ have been studied with the $Q C D$ sum rules by taking into account the perturbative contributions and the gluon condensate contributions in performing the operator product expansion [1215]. In calculations, the constant or universal heavy-quark pole masses or $\overline{M S}$ masses are chosen in all the channels [12-15]. In this article, we reexamine the mass spectrum 
of the ground state triplyheavy baryon states by taking into account the three-gluon condensates. It is for the first time to take into account the three-gluon condensates in the QCD sum rules for the triply heavy baryon states. Furthermore, we pay special attention to the heavy quark masses and choose the values which work well in studying the doubly heavy baryon states [38], hidden-charm tetraquark states [39-41], hidden-bottom tetraquark states [4244], hidden-charm pentaquark states [45-48], and fully charmed tetraquark states [49-51] to perform a novel analysis.

The article is structured as follows: In Section 2, we obtain the QCD sum rules for the masses and pole residues of the tripheavy baryon states. In Section 3, we present the numerical results and discussions. Section 4 is reserved for our conclusion.

\section{QCD sum rules for the triply heavy baryon states}

Firstly, we write down the two-point correlation functions $\Pi(p)$ and $\Pi_{\mu \nu}(p)$ in the QCD sum rules,

$$
\begin{gathered}
\Pi(p)=i \int d^{4} x e^{i p \cdot x}\langle 0|T\{J(x) \bar{J}(0)\}| 0\rangle, \\
\Pi_{\mu \nu}(p)=i \int d^{4} x e^{i p \cdot x}\left\langle 0\left|T\left\{J_{\mu}(x) \bar{J}_{v}(0)\right\}\right| 0\right\rangle,
\end{gathered}
$$

where $J(x)=J^{Q Q Q^{\prime}}(x), J_{\mu}(x)=J_{\mu}^{Q Q Q^{\prime}}(x), J_{\mu}^{Q Q Q}(x)$,

$$
\begin{aligned}
& J^{Q Q Q^{\prime}}(x)=\varepsilon^{i j k} Q_{i}^{T}(x) C \gamma_{\mu} Q_{j}(x) \gamma^{\mu} \gamma_{5} Q_{k}^{\prime}(x), \\
& J_{\mu}^{Q Q Q^{\prime}}(x)=\varepsilon^{i j k} Q_{i}^{T}(x) C \gamma_{\mu} Q_{j}(x) Q_{k}^{\prime}(x), \\
& J_{\mu}^{Q Q Q}(x)=\varepsilon^{i j k} Q_{i}^{T}(x) C \gamma_{\mu} Q_{j}(x) Q_{k}(x),
\end{aligned}
$$

where $Q, Q^{\prime}=b, c, Q \neq Q^{\prime}, i, i$ and $k$ are color indexes and $C$ is the charge conjugation matrix. We choose the Ioffe-type currents $J(x)$ and $J_{\mu}(x)$ to interpolate the triply heavy baryon states with the spin-parity $J^{P}=\frac{1}{2}^{+}$and $\frac{3}{2}^{+}$, respectively,

$$
\begin{aligned}
\left\langle 0|J(0)| \Omega_{Q Q Q^{\prime},+}(p)\right\rangle & =\lambda_{+} U_{+}(p, s), \\
\left\langle 0\left|J_{\mu}(0)\right| \Omega_{Q Q Q^{(\prime)},+}^{*}(p)\right\rangle & =\lambda_{+} U_{\mu}^{+}(p, s),
\end{aligned}
$$

where the $\Omega_{Q_{Q Q},+}$ and $\Omega_{\mathrm{QQQ}^{(1)},+}^{*}$ represent the triply heavy baryon states with the spin-parity $J^{P}=\frac{1}{2}^{+}$and $\frac{3}{2}^{+}$, respectively. $\lambda_{+}$are the pole residues, $U(p, s)$ and $U_{\mu}(p, s)$ are the Dirac spinors and the subscript or superscript + denotes the parity. The currents $J(x)$ and $J_{\mu}(x)$ also couple potentially to the negative-parity triply heavy baryon states $\Omega_{Q Q Q^{\prime},-}$ and $\Omega_{Q Q Q^{(\prime),-}}^{*}$, respectively, because multiplying $i \gamma_{5}$ to the currents $J(x)$ and $J_{\mu}(x)$ changes their parity [15, 38, 45-48, 52-59],

$$
\begin{aligned}
\left\langle 0|J(0)| \Omega_{Q Q Q^{\prime},-}(p)\right\rangle & =\lambda_{-} i \gamma_{5} U_{-}(p, s), \\
\left\langle 0\left|J_{\mu}(0)\right| \Omega_{Q Q Q^{(1)},-}^{*}(p)\right\rangle & =\lambda_{-} i \gamma_{5} U_{\mu}^{-}(p, s),
\end{aligned}
$$

again the subscript or superscript - denotes the parity. On the other hand, we can use the valance quarks and spin-parity to represent the triply heavy baryon states, for example, $Q Q Q^{\prime}\left(\frac{3}{2}^{+}\right)$. We cannot construct the currents $J^{Q Q Q}(x)=\varepsilon^{i j k} Q_{i}^{T}(x) C \gamma_{\mu} Q_{j}(x) \gamma^{\mu} \gamma_{5} Q_{k}(x)$ to interpolate the triply heavy baryon states $\Omega_{Q Q Q,+}$ with the spin-parity $J^{P}=\frac{1}{2}^{+}$because such current operators cannot exist due to the Fermi-Dirac statistics.

We insert a complete set of intermediate triply heavy baryon states with the same quantum numbers as the current operators $J(x), i \gamma_{5} J(x), J_{\mu}(x)$ and $i \gamma_{5} J_{\mu}(x)$ into the correlation functions $\Pi(p)$ and $\Pi_{\mu \nu}(p)$ to obtain the hadron representation [60-62]. After isolating the pole terms of the lowest states of the positive-parity and negative-parity triply heavy baryon states, we obtain the results:

$$
\begin{aligned}
\Pi(p) & =\lambda_{+}^{2} \frac{\not p+M_{+}}{M_{+}^{2}-p^{2}}+\lambda_{-}^{2} \frac{\not p-M_{-}}{M_{-}^{2}-p^{2}}+\cdots, \\
& =\Pi_{1}\left(p^{2}\right) \not p+\Pi_{0}\left(p^{2}\right), \\
\Pi_{\mu \nu}(p) & =\Pi_{(p)}\left(-g_{\mu \nu}+\cdots\right)+\cdots,
\end{aligned}
$$

we choose the tensor structure $g_{\mu \nu}$ to study the $\operatorname{spin} J=\frac{3}{2}$ triply heavy baryon states.

We can obtain the hadronic spectral densities $\rho_{H}^{1}(s)$ and $\rho_{H}^{0}(s)$ at the hadron side through dispersion relation,

$$
\begin{aligned}
\rho_{H}^{1}(s) & =\frac{\operatorname{Im} \Pi_{1}(s)}{\pi} \\
& =\lambda_{+}^{2} \delta\left(s-M_{+}^{2}\right)+\lambda_{-}^{2} \delta\left(s-M_{-}^{2}\right) \\
\rho_{H}^{0}(s) & =\frac{\operatorname{Im} \Pi_{0}(s)}{\pi} \\
& =M_{+} \lambda_{+}^{2} \delta\left(s-M_{+}^{2}\right)-M_{-} \lambda_{-}^{2} \delta\left(s-M_{-}^{2}\right),
\end{aligned}
$$

then introduce the weight function $\exp \left(-\frac{s}{T^{2}}\right)$ and obtain the QCD sum rules at the hadron side,

$2 M_{+} \lambda_{+}^{2} \exp \left(-\frac{M_{+}^{2}}{T^{2}}\right)=\int_{\Delta^{2}}^{s_{0}} d s\left[\sqrt{s} \rho_{H}^{1}(s)+\rho_{H}^{0}(s)\right] \exp \left(-\frac{s}{T^{2}}\right)$,

where the thresholds $\Delta^{2}=\left(2 m_{Q}+m_{Q^{\prime}}\right)^{2}$ or $9 m_{Q}^{2}$, $T^{2}$ are the Borel parameters, and $s_{0}$ are the continuum 
threshold parameters. The combinations $\sqrt{s} \rho_{H}^{1}(s)+\rho_{H}^{0}(s)$ and $\sqrt{s} \rho_{H}^{1}(s)-\rho_{H}^{0}(s)$ contain the contributions from the positive-parity and negative-parity triply heavy baryon states, respectively.

Now we briefly outline the operator product expansion performed at the deep Euclidean region $p^{2} \ll 0$. We contract the heavy quark fields in the correlation functions $\Pi(p)$ and $\Pi_{\mu \nu}(p)$ with Wick theorem, substitute the full heavy quark propagators $S_{i j}(x)$ into the correlation functions $\Pi(p)$ and $\Pi_{\mu \nu}(p)$ firstly,

$$
\begin{aligned}
S_{i j}(x)= & \frac{i}{(2 \pi)^{4}} \int d^{4} k e^{-i k \cdot x}\left\{\frac{\delta_{i j}}{k-m_{Q}}-\frac{g_{s} G_{\alpha \beta}^{n} t_{i j}^{n}}{4} \frac{\sigma^{\alpha \beta}\left(k+m_{Q}\right)+\left(k+m_{Q}\right) \sigma^{\alpha \beta}}{\left(k^{2}-m_{Q}^{2}\right)^{2}}\right. \\
& -\frac{g_{s}^{2}\left(t^{a} t^{b}\right)_{i j} G_{\alpha \beta}^{a} G_{\mu \nu}^{b}\left(f^{\alpha \beta \mu \nu}+f^{\alpha \mu \beta \nu}+f^{\alpha \mu \nu \beta}\right)}{4\left(k^{2}-m_{Q}^{2}\right)^{5}} \\
& \left.+\frac{\left\langle g_{s}^{3} G G G\right\rangle}{48} \frac{\left(k+m_{Q}\right)\left[k\left(k^{2}-3 m_{Q}^{2}\right)+2 m_{Q}\left(2 k^{2}-m_{Q}^{2}\right)\right]\left(k+m_{Q}\right)}{\left(k^{2}-m_{Q}^{2}\right)^{6}}+\cdots\right\},
\end{aligned}
$$

$f^{\alpha \beta \mu \nu}=\left(k+m_{Q}\right) \gamma^{\alpha}\left(k+m_{Q}\right) \gamma^{\beta}\left(k+m_{Q}\right) \gamma^{\mu}\left(k+m_{Q}\right) \gamma^{v}\left(k+m_{Q}\right)$,

where $\left\langle g_{s}^{3} G G G\right\rangle=\left\langle g_{s}^{3} f_{a b c} G_{\mu \nu}^{a} G_{b}^{\nu \alpha} G_{\alpha}^{b \mu}\right\rangle, t^{n}=\frac{\lambda^{n}}{2}, \lambda^{n}$ is the Gell-Mann matrix, $i, j$ are the color indexes [62], then complete the integrals in the coordinate space and momentum space sequentially to obtain the correlation functions $\Pi(p)$ and $\Pi_{\mu \nu}(p)$ at the quark-gluon level, finally we obtain the corresponding QCD spectral densities through dispersion relation,

$$
\begin{aligned}
& \rho_{Q C D}^{1}(s)=\frac{\operatorname{Im} \Pi_{1}(s)}{\pi}, \\
& \rho_{Q C D}^{0}(s)=\frac{\operatorname{Im} \Pi_{0}(s)}{\pi} .
\end{aligned}
$$

We match the hadron side with the QCD side of the correlation functions $\Pi_{1}\left(p^{2}\right)$ and $\Pi_{0}\left(p^{2}\right)$ below the continuum thresholds $s_{0}$, introduce the weight function $\exp \left(-\frac{s}{T^{2}}\right)$ and obtain the QCD sum rules,

$$
\begin{aligned}
2 M_{+} \lambda_{+}^{2} \exp \left(-\frac{M_{+}^{2}}{T^{2}}\right) & =\int_{\Delta^{2}}^{s_{0}} d s\left[\sqrt{s} \rho_{H}^{1}(s)+\rho_{H}^{0}(s)\right] \exp \left(-\frac{s}{T^{2}}\right), \\
& =\int_{\Delta^{2}}^{s_{0}} d s\left[\sqrt{s} \rho_{Q C D}^{1}(s)+\rho_{Q C D}^{0}(s)\right] \exp \left(-\frac{s}{T^{2}}\right),
\end{aligned}
$$

where $\rho_{\mathrm{QCD}}^{1}(s)=\rho_{\mathrm{QQQ}, \frac{3}{2}}^{1}(s), \rho_{\mathrm{QQQ}, \frac{3}{2}}^{1}(s), \rho_{\mathrm{QQQ}, \frac{1}{2}}^{1}(s)$, $\rho_{\mathrm{QCD}}^{0}(s)=m_{\mathrm{Q}} \rho_{\mathrm{QQQ}, \frac{3}{2}}^{0}(s), m_{Q^{\prime}} \rho_{\mathrm{QQQ}^{\prime}, \frac{3}{2}}^{0}(s), m_{Q^{\prime}} \rho_{\mathrm{QQQ}, \frac{1}{2}}^{0}(s)$,

$$
\begin{aligned}
& \rho_{\mathrm{QQQ}, \frac{3}{2}}^{1}(s) \\
& =\frac{3}{64 \pi^{4}} \int_{z_{i}}^{z_{f}} d z \int_{y_{i}}^{y_{f}} d y y z(1-y-z)\left(s-\widetilde{m}_{Q}^{2}\right)\left(11 s-5 \widetilde{m}_{Q}^{2}\right) \\
& +\frac{15 m_{Q}^{2}}{32 \pi^{4}} \int_{z_{i}}^{z_{f}} d z \int_{y_{i}}^{y_{f}} d y y\left(s-\widetilde{m}_{Q}^{2}\right) \\
& -\frac{m_{Q}^{2}}{32 \pi^{2}}\left\langle\frac{\alpha_{s} G G}{\pi}\right\rangle \int_{z_{i}}^{z_{f}} d z \int_{y_{i}}^{y_{f}} d y \frac{z(1-y-z)}{y^{2}}\left(1+\frac{3 s}{2 T^{2}}\right) \delta\left(s-\widetilde{m}_{Q}^{2}\right) \\
& -\frac{5 m_{Q}^{4}}{192 \pi^{2} T^{2}}\left\langle\frac{\alpha_{s} G G}{\pi}\right\rangle \int_{z_{i}}^{z_{f}} d z \int_{y_{i}}^{y_{f}} d y \frac{1}{y^{2}} \delta\left(s-\widetilde{m}_{Q}^{2}\right) \\
& -\frac{5 m_{Q}^{4}}{96 \pi^{2} T^{2}}\left\langle\frac{\alpha_{s} G G}{\pi}\right\rangle \int_{z_{i}}^{z_{f}} d z \int_{y_{i}}^{y_{f}} d y \frac{z}{y^{3}} \delta\left(s-\widetilde{m}_{Q}^{2}\right) \\
& +\frac{5 m_{Q}^{2}}{32 \pi^{2}}\left\langle\frac{\alpha_{s} G G}{\pi}\right\rangle \int_{z_{i}}^{z_{f}} d z \int_{y_{i}}^{y_{f}} d y \frac{z}{y^{2}} \delta\left(s-\widetilde{m}_{Q}^{2}\right) \\
& -\frac{25}{384 \pi^{2}}\left\langle\frac{\alpha_{s} G G}{\pi}\right\rangle \int_{z_{i}}^{z_{f}} d z \int_{y_{i}}^{y_{f}} d y(1-y-z)\left[1+\frac{7 s}{25} \delta\left(s-\tilde{m}_{Q}^{2}\right)\right] \\
& -\frac{5 m_{Q}^{2}}{192 \pi^{2}}\left\langle\frac{\alpha_{s} G G}{\pi}\right\rangle \int_{z_{i}}^{z_{f}} d z \int_{y_{i}}^{y_{f}} d y \frac{1}{z} \delta\left(s-\widetilde{m}_{Q}^{2}\right) \\
& -\frac{m_{Q}^{2}\left\langle g_{s}^{3} G G G\right\rangle}{512 \pi^{4} T^{2}} \int_{z_{i}}^{z_{f}} d z \int_{y_{i}}^{y_{f}} d y \frac{z(1-y-z)}{y^{3}}\left(1-\frac{3 s}{T^{2}}\right) \delta\left(s-\widetilde{m}_{Q}^{2}\right) \\
& +\frac{5 m_{Q}^{4}\left\langle g_{s}^{3} G G G\right\rangle}{768 \pi^{4} T^{4}} \int_{z_{i}}^{z_{f}} d z \int_{y_{i}}^{y_{f}} d y \frac{z}{y^{4}} \delta\left(s-\widetilde{m}_{Q}^{2}\right) \\
& +\frac{5 m_{Q}^{4}\left\langle g_{s}^{3} G G G\right\rangle}{1536 \pi^{4} T^{4}} \int_{z_{i}}^{z_{f}} d z \int_{y_{i}}^{y_{f}} d y \frac{1}{y^{3}} \delta\left(s-\widetilde{m}_{Q}^{2}\right) \\
& -\frac{\left\langle g_{s}^{3} G G G\right\rangle}{1024 \pi^{4}} \int_{z_{i}}^{z_{f}} d z \int_{y_{i}}^{y_{f}} d y \frac{z(1-y-z)}{y^{2}}\left(2+\frac{3 s}{T^{2}}\right) \delta\left(s-\widetilde{m}_{Q}^{2}\right) \\
& -\frac{5 m_{Q}^{2}\left\langle g_{s}^{3} G G G\right\rangle}{256 \pi^{4} T^{2}} \int_{z_{i}}^{z_{f}} d z \int_{y_{i}}^{y_{f}} d y \frac{z}{y^{3}} \delta\left(s-\widetilde{m}_{Q}^{2}\right) \\
& -\frac{23 m_{Q}^{2}\left\langle g_{s}^{3} G G G\right\rangle}{4608 \pi^{4} T^{2}} \int_{z_{i}}^{z_{f}} d z \int_{y_{i}}^{y_{f}} d y \frac{1}{y^{2}} \delta\left(s-\widetilde{m}_{Q}^{2}\right) \\
& +\frac{11 m_{Q}^{2}\left\langle g_{s}^{3} G G G\right\rangle}{4608 \pi^{4} T^{2}} \int_{z_{i}}^{z_{f}} d z \int_{y_{i}}^{y_{f}} d y \frac{1-y-z}{y^{2}}\left(1+\frac{7 s}{11 T^{2}}\right) \delta\left(s-\widetilde{m}_{Q}^{2}\right) \\
& +\frac{m_{Q}^{2}\left\langle g_{s}^{3} G G G\right\rangle}{2304 \pi^{4} T^{2}} \int_{z_{i}}^{z_{f}} d z \int_{y_{i}}^{y_{f}} d y \frac{1}{y}\left(1+\frac{s}{T^{2}}\right) \delta\left(s-\widetilde{m}_{Q}^{2}\right) \\
& +\frac{m_{Q}^{2}\left\langle g_{s}^{3} G G G\right\rangle}{768 \pi^{4} T^{2}} \int_{z_{i}}^{z_{f}} d z \int_{y_{i}}^{y_{f}} d y \frac{1-y-z}{z y^{2}} \delta\left(s-\widetilde{m}_{Q}^{2}\right) \\
& -\frac{m_{Q}^{2}\left\langle g_{s}^{3} G G G\right\rangle}{384 \pi^{4} T^{2}} \int_{z_{i}}^{z_{f}} d z \int_{y_{i}}^{y_{f}} d y \frac{1-y-z}{z y}\left(1-\frac{s}{3 T^{2}}\right) \delta\left(s-\tilde{m}_{Q}^{2}\right) \\
& +\frac{m_{Q}^{4}\left\langle g_{s}^{3} G G G\right\rangle}{1536 \pi^{4} T^{4}} \int_{z_{i}}^{z_{f}} d z \int_{y_{i}}^{y_{f}} d y \frac{1}{y^{3}} \delta\left(s-\widetilde{m}_{Q}^{2}\right) \\
& +\frac{5 m_{Q}^{4}\left\langle g_{s}^{3} G G G\right\rangle}{4608 \pi^{4} T^{4}} \int_{z_{i}}^{z_{f}} d z \int_{y_{i}}^{y_{f}} d y \frac{1}{z y^{2}} \delta\left(s-\widetilde{m}_{Q}^{2}\right) \\
& +\frac{m_{Q}^{4}\left\langle g_{s}^{3} G G G\right\rangle}{1536 \pi^{4} T^{4}} \int_{z_{i}}^{z_{f}} d z \int_{y_{i}}^{y_{f}} d y \frac{1-y-z}{z y^{3}} \delta\left(s-\widetilde{m}_{Q}^{2}\right) \\
& -\frac{\left\langle g_{s}^{3} G G G\right\rangle}{512 \pi^{4}} \int_{z_{i}}^{z_{f}} d z \int_{y_{i}}^{y_{f}} d y \frac{1-y-z}{y}\left(3+\frac{7 s}{6 T^{2}}\right) \delta\left(s-\widetilde{m}_{Q}^{2}\right) \\
& -\frac{5 m_{Q}^{2}\left\langle g_{s}^{3} G G G\right\rangle}{3072 \pi^{4} T^{2}} \int_{z_{i}}^{z_{f}} d z \int_{y_{i}}^{y_{f}} d y \frac{1}{z y} \delta\left(s-\widetilde{m}_{Q}^{2}\right) \\
& -\frac{m_{Q}^{2}\left\langle g_{s}^{3} G G G\right\rangle}{512 \pi^{4} T^{2}} \int_{z_{i}}^{z_{f}} d z \int_{y_{i}}^{y_{f}} d y \frac{1-y-z}{z y^{2}} \delta\left(s-\widetilde{m}_{Q}^{2}\right),
\end{aligned}
$$


Wang AAPPS Bulletin $\quad$ (2021) 31:5

Page 4 of 10

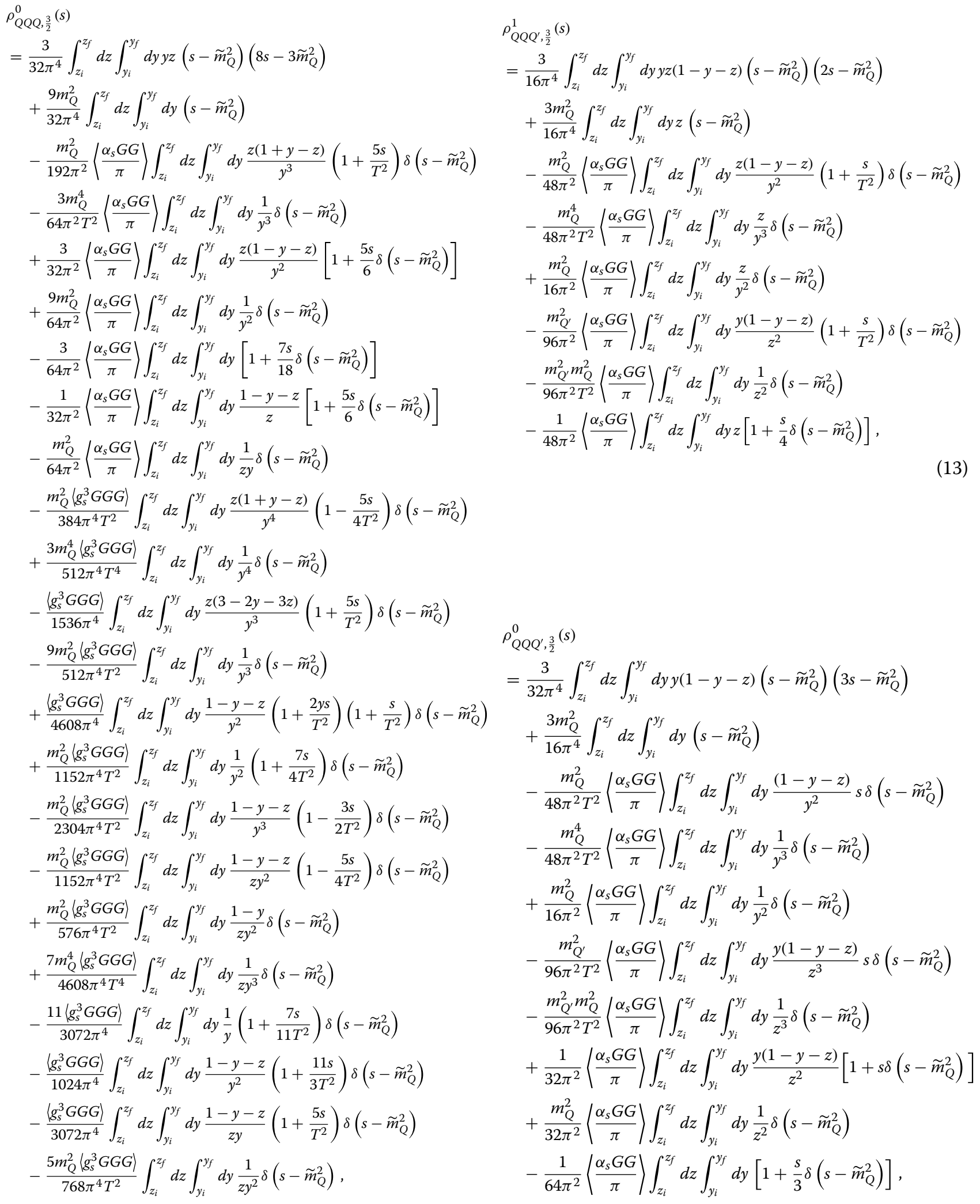

(12)

(14) 


$$
\begin{aligned}
& \rho_{Q Q Q^{\prime}, \frac{1}{2}}^{1}(s) \\
& =\frac{3}{8 \pi^{4}} \int_{z_{i}}^{z_{f}} d z \int_{y_{i}}^{y_{f}} d y y z(1-y-z)\left(s-\widetilde{m}_{Q}^{2}\right)\left(5 s-3 \widetilde{m}_{Q}^{2}\right) \\
& \quad+\frac{3 m_{Q}^{2}}{8 \pi^{4}} \int_{z_{i}}^{z_{f}} d z \int_{y_{i}}^{y_{f}} d y z\left(s-\widetilde{m}_{Q}^{2}\right) \\
& \quad-\frac{m_{Q}^{2}}{6 \pi^{2}}\left\langle\frac{\alpha_{s} G G}{\pi}\right\rangle \int_{z_{i}}^{z_{f}} d z \int_{y_{i}}^{y_{f}} d y \frac{z(1-y-z)}{y^{2}}\left(1+\frac{s}{2 T^{2}}\right) \delta\left(s-\widetilde{m}_{Q}^{2}\right) \\
& \quad-\frac{m_{Q}^{4}}{24 \pi^{2} T^{2}}\left\langle\frac{\alpha_{s} G G}{\pi}\right\rangle \int_{z_{i}}^{z_{f}} d z \int_{y_{i}}^{y_{f}} d y \frac{z}{y^{3}} \delta\left(s-\widetilde{m}_{Q}^{2}\right) \\
& \quad+\frac{m_{Q}^{2}}{8 \pi^{2}}\left\langle\frac{\alpha_{s} G G}{\pi}\right\rangle \int_{z_{i}}^{z_{f}} d z \int_{y_{i}}^{y_{f}} d y \frac{z}{y^{2}} \delta\left(s-\widetilde{m}_{Q}^{2}\right) \\
& \quad-\frac{m_{Q^{\prime}}^{2}}{12 \pi^{2}}\left\langle\frac{\alpha_{s} G G}{\pi}\right\rangle \int_{z_{i}}^{z_{f}} d z \int_{y_{i}}^{y_{f}} d y \frac{y(1-y-z)}{z^{2}}\left(1+\frac{s}{2 T^{2}}\right) \delta\left(s-\widetilde{m}_{Q}^{2}\right) \\
& \quad-\frac{m_{Q^{\prime}}^{2} m_{Q}^{2}}{48 \pi^{2} T^{2}}\left\langle\frac{\alpha_{s} G G}{\pi}\right\rangle \int_{z_{i}}^{z_{f}} d z \int_{y_{i}}^{y_{f}} d y \frac{1}{z^{2}} \delta\left(s-\widetilde{m}_{Q}^{2}\right) \\
& \quad+\frac{3}{16 \pi^{2}}\left\langle\frac{\alpha_{s} G G}{\pi}\right\rangle \int_{z_{i}}^{z_{f}} d z \int_{y_{i}}^{y_{f}} d y(1-y-z)\left[1+\frac{s}{3} \delta\left(s-\widetilde{m}_{Q}^{2}\right)\right] \\
& \quad+\frac{m_{Q}^{2}}{16 \pi^{2}}\left\langle\frac{\alpha_{s} G G}{\pi}\right\rangle \int_{z_{i}}^{z_{f}} d z \int_{y_{i}}^{y_{f}} d y \frac{1}{y} \delta\left(s-\widetilde{m}_{Q}^{2}\right),
\end{aligned}
$$

$$
\begin{aligned}
& \rho_{\mathrm{QQQ}, \frac{1}{2}}^{0}(s) \\
& =\frac{3}{8 \pi^{4}} \int_{z_{i}}^{z_{f}} d z \int_{y_{i}}^{y_{f}} d y y(1-y-z)\left(s-\widetilde{m}_{Q}^{2}\right)\left(2 s-\widetilde{m}_{Q}^{2}\right) \\
& +\frac{3 m_{Q}^{2}}{4 \pi^{4}} \int_{z_{i}}^{z_{f}} d z \int_{y_{i}}^{y_{f}} d y\left(s-\widetilde{m}_{Q}^{2}\right) \\
& -\frac{m_{Q}^{2}}{24 \pi^{2}}\left\langle\frac{\alpha_{s} G G}{\pi}\right\rangle \int_{z_{i}}^{z_{f}} d z \int_{y_{i}}^{y_{f}} d y \frac{(1-y-z)}{y^{2}}\left(1+\frac{s}{T^{2}}\right) \delta\left(s-\widetilde{m}_{Q}^{2}\right) \\
& -\frac{m_{Q}^{4}}{12 \pi^{2} T^{2}}\left\langle\frac{\alpha_{s} G G}{\pi}\right\rangle \int_{z_{i}}^{z_{f}} d z \int_{y_{i}}^{y_{f}} d y \frac{1}{y^{3}} \delta\left(s-\widetilde{m}_{Q}^{2}\right) \\
& +\frac{m_{Q}^{2}}{4 \pi^{2}}\left\langle\frac{\alpha_{s} G G}{\pi}\right\rangle \int_{z_{i}}^{z_{f}} d z \int_{y_{i}}^{y_{f}} d y \frac{1}{y^{2}} \delta\left(s-\widetilde{m}_{Q}^{2}\right) \\
& -\frac{m_{Q^{\prime}}^{2}}{48 \pi^{2}}\left\langle\frac{\alpha_{s} G G}{\pi}\right\rangle \int_{z_{i}}^{z_{f}} d z \int_{y_{i}}^{y_{f}} d y \frac{y(1-y-z)}{z^{3}}\left(1+\frac{s}{T^{2}}\right) \delta\left(s-\widetilde{m}_{Q}^{2}\right) \\
& -\frac{m_{Q^{\prime}}^{2} m_{Q}^{2}}{24 \pi^{2} T^{2}}\left\langle\frac{\alpha_{s} G G}{\pi}\right\rangle \int_{z_{i}}^{z_{f}} d z \int_{y_{i}}^{y_{f}} d y \frac{1}{z^{3}} \delta\left(s-\widetilde{m}_{Q}^{2}\right) \\
& +\frac{1}{8 \pi^{2}}\left\langle\frac{\alpha_{s} G G}{\pi}\right\rangle \int_{z_{i}}^{z_{f}} d z \int_{y_{i}}^{y_{f}} d y \frac{y(1-y-z)}{z^{2}}\left[1+\frac{s}{2} \delta\left(s-\widetilde{m}_{Q}^{2}\right)\right] \\
& +\frac{m_{Q}^{2}}{8 \pi^{2}}\left\langle\frac{\alpha_{s} G G}{\pi}\right\rangle \int_{z_{i}}^{z_{f}} d z \int_{y_{i}}^{y_{f}} d y \frac{1}{z^{2}} \delta\left(s-\widetilde{m}_{Q}^{2}\right) \\
& -\frac{1}{16 \pi^{2}}\left\langle\frac{\alpha_{s} G G}{\pi}\right\rangle \int_{z_{i}}^{z_{f}} d z \int_{y_{i}}^{y_{f}} d y\left[1+\frac{s}{2} \delta\left(s-\widetilde{m}_{Q}^{2}\right)\right] \\
& +\frac{1}{8 \pi^{2}}\left\langle\frac{\alpha_{s} G G}{\pi}\right\rangle \int_{z_{i}}^{z_{f}} d z \int_{y_{i}}^{y_{f}} d y \frac{(1-y-z)}{z}\left[1+\frac{s}{2} \delta\left(s-\widetilde{m}_{Q}^{2}\right)\right] \\
& +\frac{m_{Q}^{2}}{8 \pi^{2}}\left\langle\frac{\alpha_{s} G G}{\pi}\right\rangle \int_{z_{i}}^{z_{f}} d z \int_{y_{i}}^{y_{f}} d y \frac{1}{z y} \delta\left(s-\widetilde{m}_{Q}^{2}\right),
\end{aligned}
$$$$
\tilde{m}_{Q}^{2}=\frac{m_{Q}^{2}}{y}+\frac{m_{Q}^{2}}{1-y-z}+\frac{m_{Q^{\prime}}^{2}}{z}
$$

$$
z_{i / f}=\frac{s+m_{Q^{\prime}}^{2}-4 m_{Q}^{2} \mp \sqrt{\left(s+m_{Q^{\prime}}^{2}-4 m_{Q}^{2}\right)^{2}-4 s m_{Q^{\prime}}^{2}}}{2 s},
$$

in the case of the QQQ baryon states, we set $m_{Q^{\prime}}^{2}=m_{Q}^{2}$. When the $\delta\left(s-\tilde{m}_{Q}^{2}\right)$ functions appear, $\int_{z_{i}}^{z_{f}} d z \int_{y_{i}}^{y_{f}} d y \rightarrow$ $\int_{0}^{1} d z \int_{0}^{1-z} d y$.

We differentiate Eq. (10) with respect to $\tau=\frac{1}{T^{2}}$, then eliminate the pole residues $\lambda_{+}$and obtain the QCD sum rules for the masses of the triply heavy baryon states,

$$
M_{+}^{2}=\frac{-\frac{d}{d \tau} \int_{\Delta^{2}}^{s_{0}} d s\left[\sqrt{s} \rho_{Q C D}^{1}(s)+\rho_{Q C D}^{0}(s)\right] \exp (-s \tau)}{\int_{\Delta^{2}}^{s_{0}} d s\left[\sqrt{s} \rho_{Q C D}^{1}(s)+\rho_{Q C D}^{0}(s)\right] \exp (-s \tau)} .
$$

\section{Results and discussions}

We take the standard values of the gluon condensates $\left\langle\frac{\alpha_{s} G G}{\pi}\right\rangle=0.012 \pm 0.004 \mathrm{GeV}^{4}$ and the three-gluon condensates $\left\langle g_{s}^{3} G G G\right\rangle=0.045 \pm 0.014 \mathrm{GeV}^{6}[60-63]$ and take the $\overline{M S}$ masses of the heavy quarks $m_{c}\left(m_{c}\right)=(1.275 \pm$ $0.025) \mathrm{GeV}$ and $m_{b}\left(m_{b}\right)=(4.18 \pm 0.03) \mathrm{GeV}$ from the Particle Data Group [1], which work well in studying the doubly heavy baryon states [38], hidden-charm tetraquark states [39-41], hidden-bottom tetraquark states [4244], hidden-charm pentaquark states [45-48], and fully charmed tetraquark states [49-51] and perform a new analysis. Furthermore, we take into account the energyscale dependence of the $\overline{M S}$ masses according to the renormalization group equation,

$$
\begin{aligned}
& m_{c}(\mu)=m_{c}\left(m_{c}\right)\left[\frac{\alpha_{s}(\mu)}{\alpha_{s}\left(m_{c}\right)}\right]^{\frac{12}{33-2 n_{f}}}, \\
& m_{b}(\mu)=m_{b}\left(m_{b}\right)\left[\frac{\alpha_{s}(\mu)}{\alpha_{s}\left(m_{b}\right)}\right]^{\frac{12}{33-2 n_{f}}}, \\
& \alpha_{s}(\mu)=\frac{1}{b_{0} t}\left[1-\frac{b_{1}}{b_{0}^{2}} \frac{\log t}{t}+\frac{b_{1}^{2}\left(\log ^{2} t-\log t-1\right)+b_{0} b_{2}}{b_{0}^{4} t^{2}}\right],
\end{aligned}
$$

where $t=\log \frac{\mu^{2}}{\Lambda^{2}}, b_{0}=\frac{33-2 n_{f}}{12 \pi}, b_{1}=\frac{153-19 n_{f}}{24 \pi^{2}}, b_{2}=$ $\frac{2857-\frac{5033}{9} n_{f}+\frac{325}{27} n_{f}^{2}}{128 \pi^{3}}, \Lambda=210 \mathrm{MeV}, 292 \mathrm{MeV}$, and $332 \mathrm{MeV}$ for the flavors $n_{f}=5,4$ and 3, respectively [1]. For the $c c b, b b c$, and $b b b$ baryon states, we choose the flavor numbers $n_{f}=5$, for the $c c c$ baryon state, we choose the flavor numbers $n_{f}=4$, and choose the optimal energy scales $\mu$ to obtain stable QCD sum rules in different channels to enhance the pole contributions in a consistent way, while in previous works, the heavy quark masses were just taken 
Table 1 The Borel windows, continuum threshold parameters, energy scales of the QCD spectral densities, pole contributions, masses, and pole residues for the triply heavy baryon states

\begin{tabular}{lllllll}
\hline & $T^{2}\left(\mathrm{GeV}^{2}\right)$ & $\sqrt{s_{0}}(\mathrm{GeV})$ & $\mu(\mathrm{GeV})$ & pole & $M(\mathrm{GeV})$ & $\lambda\left(10^{-1} \mathrm{GeV}^{3}\right)$ \\
\hline$c c c\left(\frac{3}{2}^{+}\right)$ & $3.1-4.1$ & $5.35 \pm 0.10$ & 1.2 & $(56-83) \%$ & $4.81 \pm 0.10$ & $(2.08 \pm 0.31)$ \\
$c c b\left(\frac{3}{2}^{+}\right)$ & $4.7-5.7$ & $8.55 \pm 0.10$ & 2.1 & $(65-85) \%$ & $8.03 \pm 0.08$ & $(2.25 \pm 0.25)$ \\
$c c b\left(\frac{1}{2}^{+}\right)$ & $4.9-5.9$ & $8.55 \pm 0.10$ & 2.0 & $(64-84) \%$ & $8.02 \pm 0.08$ & $(4.30 \pm 0.47)$ \\
$b b c\left(\frac{3}{2}^{+}\right)$ & $6.4-7.4$ & $11.75 \pm 0.10$ & 2.2 & $(65-83) \%$ & $11.23 \pm 0.08$ & $(3.24 \pm 0.46)$ \\
$b b c\left(\frac{1}{2}^{+}\right)$ & $6.3-7.3$ & $11.75 \pm 0.10$ & 2.2 & $(65-84) \%$ & $11.22 \pm 0.08$ & $(5.65 \pm 0.81)$ \\
$b b b\left(\frac{3}{2}^{+}\right)$ & $8.6-9.6$ & $14.95 \pm 0.10$ & 2.5 & $(66-83) \%$ & $14.43 \pm 0.09$ & $(9.42 \pm 1.39)$ \\
\hline
\end{tabular}

as mass parameters, had constant values in all the QCD sum rules [12-15].

As long as the continuum threshold parameters $s_{0}$ are concerned, we should choose suitable values to avoid contaminations from the first radial excited states of the triply heavy baryon states and can borrow some ideas from the experimental data on the conventional charmonium (bottomonium) states and the charmonium-like states. The energy gaps between the ground states and the first radial excited states are $M_{\psi^{\prime}}-M_{J / \psi}=589 \mathrm{MeV}$ and $M_{\Upsilon^{\prime}}-M_{\Upsilon}=563 \mathrm{MeV}$ from the Particle Data Group [1], $M_{B_{c}^{* \prime}}-M_{B_{c}^{*}}=567 \mathrm{MeV}$ from the CMS collaboration [64], $M_{B_{c}^{* \prime}}-M_{B_{c}^{*}}=566 \mathrm{MeV}$ from the LHCb collaboration [65], $M_{Z_{c}(4430)}-M_{Z_{c}(3900)}=591 \mathrm{MeV}, M_{X(4500)}-M_{X(3915)}=$ $588 \mathrm{MeV}$ from the Particle Data Group [1], $M_{Z_{c}(4600)}-$ $M_{Z_{c}(4020)}=576 \mathrm{MeV}$ from the LHCb collaboration [66]. We usually assign the $Z_{c}^{ \pm}$(3900) and $Z_{c}^{ \pm}$(4430) to be the ground state and the first radial excited state of the axialvector tetraquark states respectively [67-69], assign the $X(3915)$ and $X(4500)$ to be the ground state and first radial excited state of the scalar tetraquark states respec- tively [70-72], and assign the $Z_{c}^{ \pm}(4020)$ and $Z_{c}^{ \pm}(4600)$ to be the ground state and first radial excited state of the axialvector tetraquark states respectively with different quark structures from that of the $Z_{c}^{ \pm}(3900)$ and $Z_{c}^{ \pm}$(4430) $[39,73,74]$.

In the present work, we can take the experimental data from the Particle Data Group, CMS, and LHCb collaborations as input parameters and choose the continuum threshold parameters as $\sqrt{s_{0}}=M_{\Omega / \Omega^{*}}+(0.50 \sim$ $0.55) \mathrm{GeV}$ as a constraint to study the triply heavy baryon states with the QCD sum rules. Furthermore, we add an uncertainty $\delta \sqrt{s_{0}}= \pm 0.1 \mathrm{GeV}$ as we usually do in estimating the uncertainties from the continuum threshold parameters in the QCD sum rules. We vary the energy scales of the QCD spectral densities, the continuum threshold parameters, and the Borel parameters to satisfy two basic criteria of the QCD sum rules, i.e., the ground state dominance at the hadron side and the operator product expansion convergence at the QCD side. After trial and error, we obtain the ideal energy scales of the QCD spectral densities, the Borel parameters $T^{2}$,
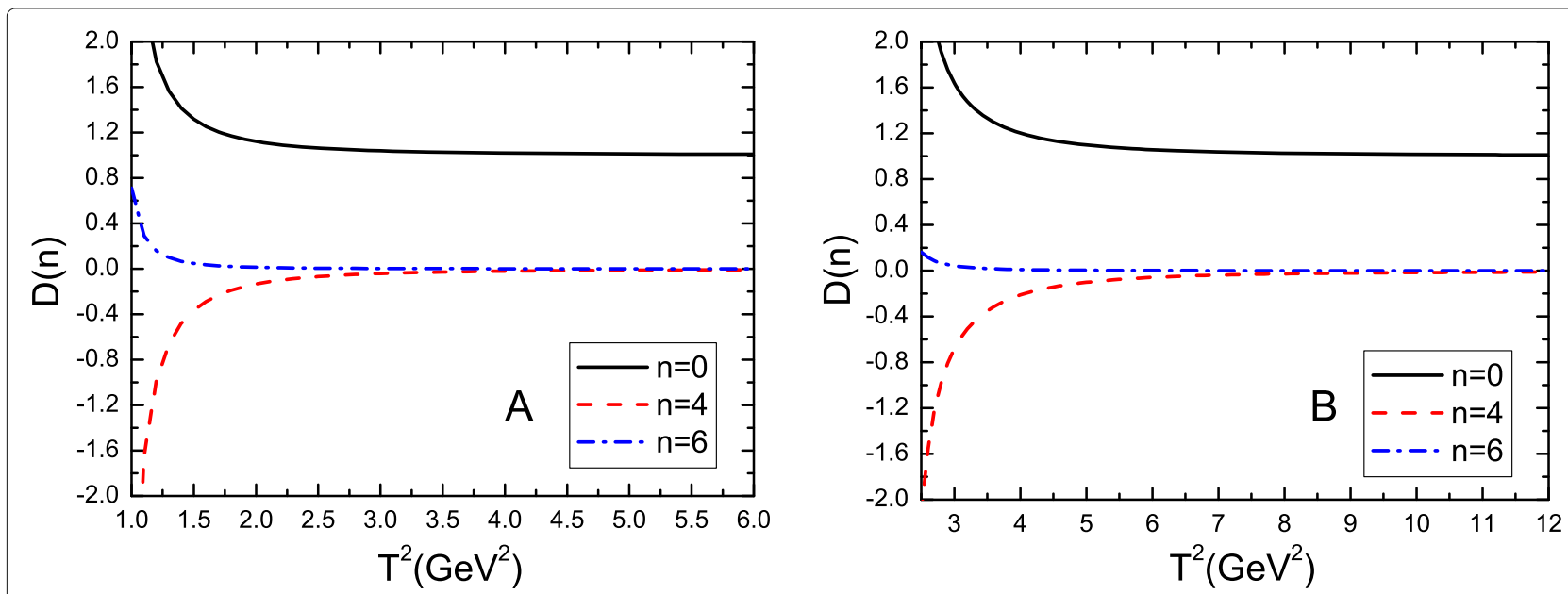

Fig. 1 The contributions of the vacuum condensates with variations of the Borel parameters $T^{2}$, where $A$ and $B$ denote the baryon states $c c c\left(\frac{3}{2}^{+}\right)$ and $b b b\left(\frac{3}{2}^{+}\right)$, respectively, $n=0,4,6$ denotes dimensions of the vacuum condensates 
and the continuum threshold parameters $s_{0}$, therefore the pole contributions of the ground state triply heavy baryon states; see Table 1.
In the Borel windows, the pole contributions are about $(60-80) \%$ and the pole dominance is well satisfied. In Fig. 1, we plot the contributions of the perturbative terms,
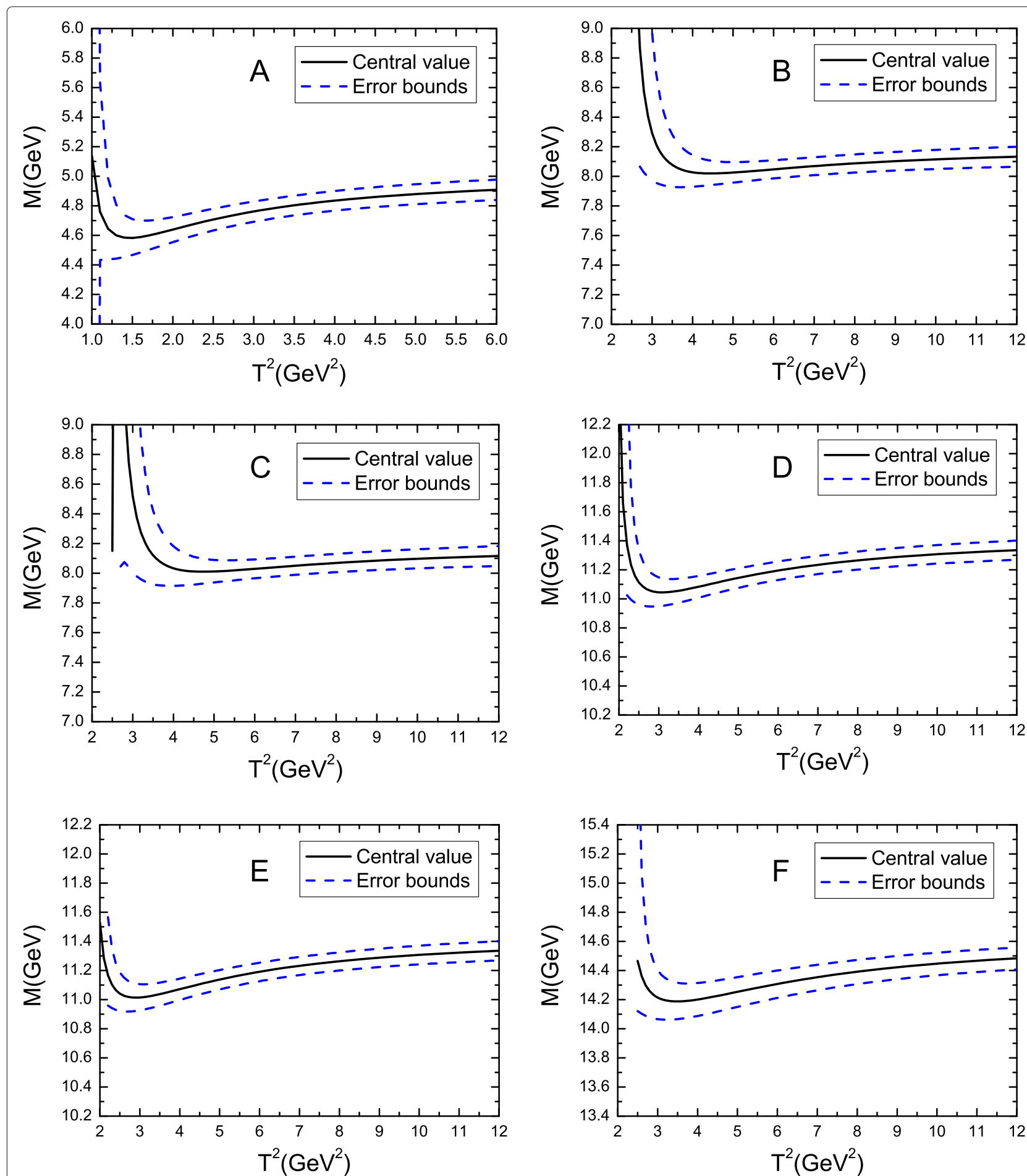

Fig. 2 The masses of the triply heavy baryon states with variations of the Borel parameters $T^{2}$, where $A, B, C, D, E$, and $F$ denote the baryon states $c c c\left(\frac{3}{2}^{+}\right), c c b\left(\frac{3}{2}^{+}\right), c c b\left(\frac{1}{2}^{+}\right), b b c\left(\frac{3}{2}^{+}\right), b b c\left(\frac{1}{2}^{+}\right)$, and $b b b\left(\frac{3}{2}^{+}\right)$, respectively 
the gluon condensates, and the three-gluon condensates with variations of the Borel parameters for the central values of the continuum threshold parameters shown in Table 1 in the QCD sum rules for the $c c c$ and $b b b$ baryon states. From the figure, we can see that the main contributions come from the perturbative terms, the gluon condensates play less important role, and the three-gluon condensates play a tiny role in the Borel windows. The Borel parameters have the relation $T_{b b b}^{2}>T_{b b c}^{2}>T_{c c b}^{2}>$
$T_{c c c}^{2}$. We add the subscripts $b b b, b b c, c c b$, and $c c c$ to denote the corresponding QCD sum rules. From Fig. 1, we can see that the contributions from the three-gluon condensates decrease quickly with the increase of the Borel parameters, at the region $T^{2} \geq 1.5 \mathrm{GeV}^{2}$ in the $c c c$ channel and at the region $T^{2} \geq 3.0 \mathrm{GeV}^{2}$ in the $b b b$ channel, the contributions from the three-gluon condensates reach zero and can be neglected safely. On the other hand, the Borel window $T_{c c c}^{2}>3.0 \mathrm{GeV}^{2}$, so we can neglect the three-gluon

Table 2 The masses of the triply heavy baryon states from different theoretical approaches, where the unit is GeV

\begin{tabular}{|c|c|c|c|c|c|c|}
\hline & $\operatorname{ccc}\left(\frac{3}{2}^{+}\right)$ & $c c b\left(\frac{3}{2}^{+}\right)$ & $\operatorname{ccb}\left(\frac{1}{2}^{+}\right)$ & $b b c\left(\frac{3}{2}^{+}\right)$ & $b b c\left(\frac{1}{2}^{+}\right)$ & $b b b\left(\frac{3}{2}^{+}\right)$ \\
\hline This Work & $4.81 \pm 0.10$ & $8.03 \pm 0.08$ & $8.02 \pm 0.08$ & $11.23 \pm 0.08$ & $11.22 \pm 0.08$ & $14.43 \pm 0.09$ \\
\hline [4] & 4.763 & & & & & \\
\hline [5] & 4.796 & 8.037 & 8.007 & 11.229 & 11.195 & 14.366 \\
\hline [6] & 4.769 & & & & & \\
\hline [7] & 4.789 & & & & & \\
\hline [8] & 4.761 & & & & & \\
\hline [9] & 4.734 & & & & & \\
\hline [10] & & & & & & 14.371 \\
\hline [11] & & 8.026 & 8.005 & 11.211 & 11.194 & \\
\hline [12] & $4.67 \pm 0.15$ & $7.45 \pm 0.16$ & $7.41 \pm 0.13$ & $10.54 \pm 0.11$ & $10.30 \pm 0.10$ & $13.28 \pm 0.10$ \\
\hline [13] & $4.72 \pm 0.12$ & $8.07 \pm 0.10$ & & $11.35 \pm 0.15$ & & $14.30 \pm 0.20$ \\
\hline [14] & & & $8.50 \pm 0.12$ & & $11.73 \pm 0.16$ & \\
\hline [15] & $4.99 \pm 0.14$ & $8.23 \pm 0.13$ & $8.23 \pm 0.13$ & $11.49 \pm 0.11$ & $11.50 \pm 0.11$ & $14.83 \pm 0.10$ \\
\hline [16] & 4.965 & 8.265 & 8.245 & 11.554 & 11.535 & 14.834 \\
\hline [17] & 4.798 & 8.023 & 8.004 & 11.221 & 11.200 & 14.396 \\
\hline [18] & 4.763 & & & & & 14.371 \\
\hline [19] & 4.760 & 8.032 & 7.999 & 11.287 & 11.274 & 14.370 \\
\hline [20] & 4.799 & 8.019 & & 11.217 & & 14.398 \\
\hline [21] & 4.76 & 7.98 & 7.98 & 11.19 & 11.19 & 14.37 \\
\hline [22] & 4.777 & 8.005 & 7.984 & 11.163 & 11.139 & 14.276 \\
\hline [23] & 4.79 & 8.03 & & 11.20 & & 14.30 \\
\hline [24] & 4.803 & 8.025 & 8.018 & 11.287 & 11.280 & 14.569 \\
\hline [25] & 4.806 & & & & & 14.496 \\
\hline [26] & 4.897 & 8.273 & 8.262 & 11.589 & 11.546 & 14.688 \\
\hline [27] & 4.773 & & & & & \\
\hline [28] & 4.828 & & & & & 14.432 \\
\hline [29] & 4.900 & 8.140 & & 10.890 & & 14.500 \\
\hline [30] & 4.799 & 8.046 & 8.018 & 11.245 & 11.214 & 14.398 \\
\hline [31] & 4.798 & & 8.018 & & 11.215 & 14.398 \\
\hline [32] & 4.760 & 7.963 & 7.867 & 11.167 & 11.077 & 14.370 \\
\hline [33] & 4.799 & & & & & 14.244 \\
\hline [34] & 5.00 & & 8.19 & & & 14.57 \\
\hline [35] & 4.93 & 8.03 & 8.01 & 11.12 & 11.09 & 14.23 \\
\hline$[36]$ & 4.834 & & & & & \\
\hline [37] & & & & & & 14.788 \\
\hline
\end{tabular}


condensates in the QCD sum rules for the $c c b$ and $b b c$ baryon states without impairing the predictive ability. The operator product expansion is well convergent. Although the three-gluon condensates play a tiny role in the Borel windows and can be neglected in the Borel windows, we take them into account to obtain the values $T^{2} \geq 1.5 \mathrm{GeV}^{2}$ or $T^{2} \geq 3.0 \mathrm{GeV}^{2}$, the calculations are non-trivial.

Now we take into account all uncertainties of the input parameters and obtain the values of the masses and pole residues of the triply heavy baryon states, which are shown explicitly in Table 1 and Fig. 2. In Fig. 2, we plot the masses of the triply heavy baryon states with variations of the Borel parameters $T^{2}$ in much larger ranges than the Borel windows. From the figure, we can see that there appear platforms in the Borel windows indeed, the uncertainties originate from the Borel parameters are very small and it is reliable to extract the triply heavy baryon masses.

In Table 2, we also present the predictions of the triply heavy baryon masses from the lattice QCD [4-11], the QCD sum rules [12-15], various potential quark models [16-31], the Fadeev equation [32-35], and the Regge trajectories [36, 37]. From the table, we can see that the predicted masses are $M_{c c c, \frac{3}{2}}{ }^{+}=(4.7 \sim 5.0) \mathrm{GeV}, M_{c c b, \frac{3}{2}^{+}}=$ $(7.5 \sim 8.3) \mathrm{GeV}, M_{c c b, \frac{1}{2}}^{+}=(7.4 \sim 8.5) \mathrm{GeV}, M_{b b c, \frac{3}{2}^{+}}=$ $(10.5 \sim 11.6) \mathrm{GeV}, M_{b b c, \frac{1}{2}}{ }^{+}=(10.3 \sim 11.7) \mathrm{GeV}$, $M_{b b b, \frac{3}{2}}{ }^{+}=(13.3 \sim 14.8) \mathrm{GeV}$ from the previous works, the present predictions $M_{c c c, \frac{3}{2}}{ }^{+}=(4.81 \pm 0.10) \mathrm{GeV}$, $M_{c c b, \frac{3}{2}}{ }^{+}=(8.03 \pm 0.08) \mathrm{GeV}, M_{c c b, \frac{1}{2}}{ }^{+}=(8.02 \pm 0.08) \mathrm{GeV}$, $M_{b b c, \frac{3}{2}}{ }^{+}=(11.23 \pm 0.08) \mathrm{GeV}, M_{b b c, \frac{1}{2}^{+}}=(11.22 \pm$ $0.08) \mathrm{GeV}, M_{b b b, \frac{3}{2}}{ }^{+}=(14.43 \pm 0.09) \mathrm{GeV}$, which are compatible with them, but with refined and more robust values compared to the previous calculations based on the QCD sum rules [12-15].

\section{Conclusion}

In this article, we reexamine the ground state triply heavy baryon states with the QCD sum rules by carrying out the operator product expansion up to the vacuum condensates of dimension 6 in a consistent way and performing a novel analysis. It is for the first time to take into account the three-gluon condensates in the QCD sum rules for the triply heavy baryon states. In calculations, we choose the $\overline{M S}$ masses of the heavy quarks which work well in studying the doubly heavy baryon states, hidden-charm tetraquark states, hidden-bottom tetraquark states, hidden-charm pentaquark states, fully charmed tetraquark states, and vary the energy scales to select the optimal values so as to obtain more stable QCD sum rules and enhance the pole contributions. The present predictions of the triply heavy baryon masses are compatible with the existing theoretical calculations but with refined and more robust values compared to the pre- vious calculations based on the QCD sum rules, which can be confronted to the experimental data in the future to make contributions to the mass spectrum of the heavy baryon states.

\section{Acknowledgements}

This work is supported by National Natural Science Foundation, Grant Number 11775079 .

\section{Authors' contributions}

The author read and approved the final manuscript.

\section{Competing interests}

The author declares that he has no competing interests.

Received: 22 November 2020 Accepted: 12 January 2021

Published online: 19 February 2021

References

1. P. A. Zyla, et al., Review of Particle Physics. Prog. Theor. Exp. Phys. 2020 083C01 (2020)

2. R. Aaij, et al., Observation of the doubly charmed baryon $\boldsymbol{\Xi}_{c c}^{++}$. Phys. Rev. Lett. 119, 112001 (2017)

3. R. Aaij, et al., Observation of structure in the $J / \psi$-pair mass spectrum. arXiv:2006.16957

4. M. Padmanath, R. G. Edwards, N. Mathur, M. Peardon, Spectroscopy of triply-charmed baryons from lattice QCD. Phys. Rev. D90, 074504 (2014)

5. Z. S. Brown, W. Detmold, S. Meinel, K. Orginos, Charmed bottom baryon spectroscopy from lattice QCD. Phys. Rev. D90, 094507 (2014)

6. K. U. Can, G. Erkol, M. Oka, T. T. Takahashi, Look inside charmed-strange baryons from lattice QCD. Phys. Rev. D92, 114515 (2015)

7. Y. Namekawa, et al., Charmed baryons at the physical point in $2+1$ flavor lattice QCD. Phys. Rev. D87, 094512 (2013)

8. R. A. Briceno, H. W. Lin, D. R. Bolton, Charmed-Baryon Spectroscopy from Lattice QCD with $N_{f}=2+1+1$ Flavors. Phys. Rev. D86, 094504 (2012)

9. C. Alexandrou, V. Drach, K. Jansen, C. Kallidonis, G. Koutsou, Baryon spectrum with $N_{f}=2+1+1$ twisted mass fermions. Phys. Rev. D90, 074501 (2014)

10. S. Meinel, Excited-state spectroscopy of triply-bottom baryons from lattice QCD. Phys. Rev. D85, 114510 (2012)

11. N. Mathur, M. Padmanath, S. Mondal, Precise predictions of charmed-bottom hadrons from lattice QCD. Phys. Rev. Lett. 121, 202002 (2018)

12. J. R. Zhang, M. Q. Huang, Deciphering triply heavy baryons in terms of QCD sum rules. Phys. Lett. B674, 28 (2009)

13. T. M. Aliev, K. Azizi, M. Savci, Properties of triply heavy spin-3/2 baryons. J. Phys. G41, 065003 (2014)

14. T. M. Aliev, K. Azizi, M. Savci, Masses and Residues of the Triply Heavy Spin-1/2 Baryons. JHEP. 1304, 042 (2013)

15. Z. G. Wang, Analysis of the Triply Heavy Baryon States with QCD Sum Rules. Commun. Theor. Phys. 58, 723 (2012)

16. W. Roberts, M. Pervin, Heavy baryons in a quark model. Int. J. Mod. Phys. A23, 2817 (2008)

17. G. Yang, J. Ping, P. G. Ortega, J. Segovia, Triply heavy baryons in the constituent quark model. Chin. Phys. C44, 023102 (2020)

18. J. Vijande, A. Valcarce, H. Garcilazo, Constituent-quark model description of triply heavy baryon nonperturbative lattice QCD data. Phys. Rev. D91, 054011 (2015)

19. K. Thakkar, A. Majethiya, P. C. Vinodkumar, Magnetic moments of baryons containing all heavy quarks in the quark-diquark model. Eur. Phys. J. Plus. 131, 339 (2016)

20. B. Silvestre-Brac, Spectrum and static properties of heavy baryons. Few Body Syst. 20, 1 (1996)

21. Y. Jia, Variational study of weakly coupled triply heavy baryons. JHEP. 0610, 073 (2006)

22. A. Bernotas, V. Simonis, Heavy hadron spectroscopy and the bag model. Lith. J. Phys. 49, 19 (2009)

23. P. Hasenfratz, R. R. Horgan, J. Kuti, J. M. Richard, Heavy Baryon Spectroscopy in the QCD Bag Model. Phys. Lett. 94B, 401 (1980) 
24. A. P. Martynenko, Ground-state triply and doubly heavy baryons in a relativistic three-quark model. Phys. Lett. B663, 317 (2008)

25. Z. Shah, A. K. Rai, Masses and Regge trajectories of triply heavy $\Omega_{c c c}$ and $\Omega_{b b b}$ baryons. Eur. Phys. J. A53, 195 (2017)

26. B. Patel, A. Majethiya, P. C. Vinodkumar, Masses and Magnetic moments of Triply Heavy Flavour Baryons in Hypercentral Model. Pramana. 72, 679 (2009)

27. S. Migura, D. Merten, B. Metsch, H. R. Petry, Charmed baryons in a relativistic quark model. Eur. Phys. J. A28, 41 (2006)

28. M. S. Liu, Q. F. Lu, X. H. Zhong, Triply charmed and bottom baryons in a constituent quark model. Phys. Rev. D101, 074031 (2020)

29. F. J. Llanes-Estrada, O. I. Pavlova, R. Williams, A First Estimate of Triply Heavy Baryon Masses from the pNRQCD Perturbative Static Potential. Eur. Phys. J. C72, 2019 (2012)

30. J. M. Flynn, E. Hernandez, J. Nieves, Triply Heavy Baryons and Heavy Quark Spin Symmetry. Phys. Rev. D85, 014012 (2012)

31. M. C. Gordillo, F. De Soto, J. Segovia, Diffusion Monte Carlo calculations of fully-heavy multiquark bound states. arXiv:2009.11889

32. S. X. Qin, C. D. Roberts, S. M. Schmidt, Spectrum of light- and heavy-baryons. Few Body Syst. 60, 26 (2019)

33. M. Radin, S. Babaghodrat, M. Monemzadeh, Estimation of heavy baryon masses $\Omega_{c c c}^{++}$and $\Omega_{b b b}^{-}$by solving the Faddeev equation in a three-dimensional approach. Phys. Rev. D90, 047701 (2014)

34. P. L. Yin, C. Chen, G. Krein, C. D. Roberts, J. Segovia, S. S. Xu, Masses of ground-state mesons and baryons, including those with heavy quarks. Phys. Rev. D100, 034008 (2019)

35. L. X. Gutierrez-Guerrero, A. Bashir, M. A. Bedolla, E. Santopinto, Masses of Light and Heavy Mesons and Baryons: A Unified Picture. Phys. Rev. D100 $114032(2019)$

36. K. W. Wei, B. Chen, X. H. Guo, Masses of doubly and triply charmed baryons. Phys. Rev. D92, 076008 (2015)

37. K. W. Wei, B. Chen, N. Liu, Q. Q. Wang, X. H. Guo, Spectroscopy of singly, doubly, and triply bottom baryons. Phys. Rev. D95, 116005 (2017)

38. Z. G. Wang, Analysis of the doubly heavy baryon states and pentaquark states with QCD sum rules. Eur. Phys. J. C78, 826 (2018)

39. Z. G. Wang, Analysis of the hidden-charm tetraquark mass spectrum with the QCD sum rules. Phys. Rev. D102, 014018 (2020)

40. Z. G. Wang, Lowest vector tetraquark states: $Y(4260 / 4220)$ or $Z_{c}(4100)$ Eur. Phys. J. C78, 933 (2018)

41. Z. G. Wang, Analysis of the vector tetraquark states with P-waves between the diquarks and antidiquarks via the QCD sum rules. Eur. Phys. J. C79, 29 (2019)

42. Z. G. Wang, T. Huang, The $Z_{b}(10610)$ and $Z_{b}(10650)$ as axial-vector tetraquark states in the QCD sum rules. Nucl. Phys. A930, 63 (2014)

43. Z. G. Wang, Analysis of the hidden-bottom tetraquark mass spectrum with the QCD sum rules, (2019), p. 489

44. Z. G. Wang, Vector hidden-bottom tetraquark candidate: $Y(10750)$. Chin. Phys. C43, 123102 (2019)

45. Z. G. Wang, Analysis of $P_{c}(4380)$ and $P_{c}(4450)$ as pentaquark states in the diquark model with QCD sum rules. Eur. Phys. J. C76, 70 (2016)

46. Z. G. Wang, T. Huang, Analysis of the $\frac{1}{2}^{ \pm}$pentaquark states in the diquark model with QCD sum rules. Eur. Phys. J. C76, 43 (2016)

47. Z. G. Wang, Analysis of the $\frac{3}{2}^{ \pm}$pentaquark states in the diquark-diquark-antiquark model with QCD sum rules. Nucl. Phys. B913 163 (2016)

48. Z. G. Wang, Analysis of the $P_{c}(4312), P_{c}(4440), P_{c}(4457)$ and related hidden-charm pentaquark states with QCD sum rules. Int. J. Mod. Phys. A35, 2050003 (2020)

49. Z. G. Wang, Analysis of the $Q Q \bar{Q} \bar{Q}$ tetraquark states with $Q C D$ sum rules. Eur. Phys. J. C77, 432 (2017)

50. Z. G. Wang, Z. Y. Di, Analysis of the vector and axialvector $Q Q \bar{Q} \bar{Q}$ tetraquark states with QCD sum rules. Acta Phys. Polon. B50, 1335 (2019)

51. Z. G. Wang, Tetraquark candidates in the LHCb's di- $J / \psi$ mass spectrum. Chin. Phys. C44, 113106 (2020)

52. Y. Chung, H. G. Dosch, M. Kremer, D. Schall, Baryon Sum Rules and Chiral Symmetry Breaking. Nucl. Phys. B197, 55 (1982)

53. E. Bagan, M. Chabab, H. G. Dosch, S. Narison, Baryon sum rules in the heavy quark effective theory. Phys. Lett. B301, 243 (1993)

54. D. Jido, N. Kodama, M. Oka, Negative parity nucleon resonance in the QCD sum rule. Phys. Rev. D54, 4532 (1996)

55. Z. G. Wang, Reanalysis of the heavy baryon states $\Omega_{b}, \Omega_{c}, \mathbf{\Xi}_{b^{\prime}}^{\prime} \mathbf{\Xi}_{c^{\prime}}^{\prime} \Sigma_{b}$ and $\Sigma_{c}$ with QCD sum rules. Phys. Lett. pages (2010)
56. Z. G. Wang, Analysis of the $\frac{1}{2}^{+}$doubly heavy baryon states with QCD sum rules. Eur. Phys. J. A45, 267 (2010)

57. Z. G. Wang, Analysis of the $\frac{3}{2}^{+}$heavy and doubly heavy baryon states with QCD sum rules. Eur. Phys. J. C68, 459 (2010)

58. Z. G. Wang, Analysis of the $\frac{1}{2}^{-}$and $\frac{3}{2}^{-}$heavy and doubly heavy baryon states with QCD sum rules. Eur. Phys. J. A47, 81 (2011)

59. Z. G. Wang, Analysis of $\Omega_{c}(3000), \Omega_{c}(3050), \Omega_{c}(3066), \Omega_{c}(3090)$ and $\Omega_{c}(3119)$ with QCD sum rules. Eur. Phys. J. C77, 325 (2017)

60. M. A. Shifman, A. I. Vainshtein, V. I. Zakharov, QCD and Resonance Physics: Theoretical Foundations. Nucl. Phys. B147, 385 (1979)

61. M. A. Shifman, A. I. Vainshtein, V. I. Zakharov, QCD and Resonance Physics: Applications. Nucl. Phys. B147, 448 (1979)

62. L. J. Reinders, H. Rubinstein, S. Yazaki, Hadron Properties from QCD Sum Rules. Phys. Rept. 127, 1 (1985)

63. P. Colangelo, A. Khodjamirian, QCD sum rules, a modern perspective. hep-ph/0010175

64. A. M. Sirunyan, et al., Observation of Two Excited $B_{c}^{+}$States and Measurement of the $B_{c}^{+}(2 \mathrm{~S})$ in $p p$ Collisions at $\sqrt{s}=13 \mathrm{TeV}$. Phys. Rev. Lett. 122, 132001 (2019)

65. R. Aaij, et al., Observation of an excited $B_{c}^{+}$state. Phys. Rev. Lett. 122, 232001 (2019)

66. R. Aaij, et al., Model-Independent Observation of Exotic Contributions to $B^{0} \rightarrow J / \psi K^{+} \pi^{-}$Decays. Phys. Rev. Lett. 122, 152002 (2019)

67. L. Maiani, F. Piccinini, A. D. Polosa, V. Riquer, The $Z(4430)$ and a New Paradigm for Spin Interactions in Tetraquarks. Phys. Rev. D89, 114010 (2014)

68. M. Nielsen, F. S. Navarra, Charged Exotic Charmonium States. Mod. Phys. Lett. A29, 1430005 (2014)

69. Z. G. Wang, Analysis of the $Z$ (4430) as the first radial excitation of the $Z_{c}$ (3900). Commun. Theor. Phys. 63, 325 (2015)

70. R. F. Lebed, A. D. Polosa, $\chi_{c 0}$ (3915) As the Lightest $c \bar{c} s \bar{s}$ State. Phys. Rev. D93, 094024 (2016)

71. Z. G. Wang, Scalar tetraquark state candidates: $X(3915), X(4500)$ and X(4700). Eur. Phys. J. C77, 78 (2017)

72. Z. G. Wang, Reanalysis of the $X(3915), X(4500)$ and $X(4700)$ with QCD sum rules. Eur. Phys. J. A53, 19 (2017)

73. H. X. Chen, W. Chen, Settling the $Z_{c}(4600)$ in the charged charmoniumlike family. Phys. Rev. D99, 074022 (2019)

74. Z. G. Wang, Axialvector tetraquark candidates for $Z_{c}(3900), Z_{c}(4020)$, $Z_{c}(4430), Z_{c}(4600)$. Chin. Phys. C44, 063105 (2020)

\section{Publisher's Note}

Springer Nature remains neutral with regard to jurisdictional claims in published maps and institutional affiliations.

\section{Submit your manuscript to a SpringerOpen ${ }^{\circ}$ journal and benefit from:}

- Convenient online submission

- Rigorous peer review

- Open access: articles freely available online

- High visibility within the field

- Retaining the copyright to your article

Submit your next manuscript at springeropen.com 